Small-time ruin for a financial process modulated by a Harris recurrent Markov chain.

Collamore, Jeffrey F.; Hoeing, A.

Published in:

Finance and Stochastics

Publication date:

2007

Document version

Publisher's PDF, also known as Version of record

Citation for published version (APA):

Collamore, J. F., \& Hoeing, A. (2007). Small-time ruin for a financial process modulated by a Harris recurrent Markov chain. Finance and Stochastics, 11(3), 299-322. 


\title{
Small-time ruin for a financial process modulated by a Harris recurrent Markov chain
}

\author{
Jeffrey F. Collamore • Andrea Höing
}

Received: 23 May 2005 / Accepted: 17 April 2007 /

Published online: 26 May 2007

(C) Springer-Verlag 2007

\begin{abstract}
We consider a nonstandard ruin problem where: (i) the increments of the process are heavy-tailed and Markov-dependent, modulated by a general Harris recurrent Markov chain; (ii) ruin occurs when a positive boundary is attained within a sufficiently small time. Our main result provides sharp asymptotics for the small-time probability of ruin, viz., $\mathbf{P}\left(\sup _{n \leq \delta u} S_{n} \geq u\right)$, where $\left\{S_{n}\right\}$ denotes the discrete partial sums of the process and $\delta \in(0,1 / \mu)$, where $\mu$ is the mean drift. We apply our results to obtain risk estimates which quantify, e.g., repetitive operational risk losses or the extremal behavior for a $\operatorname{GARCH}(1,1)$ process.
\end{abstract}

Keywords Ruin probabilities · Large deviations · Subexponential distributions · GARCH processes $\cdot$ Repetitive operational risk modeling

\section{JEL Classification G10}

Mathematics Subject Classification (2000) 60G70 - 60J10 • 60F10

Research supported in part by Danish Research Council (SNF) Grant 21-04-0400. Parts of this work are contained in A. Höing's Ph.D. thesis, written at ETH Zürich under the supervision of Prof. P. Embrechts. This work was begun while J.F. Collamore was a post-doctoral research fellow at RiskLab, ETH Zürich.

J.F. Collamore $(\bowtie)$

Department of Mathematical Sciences, University of Copenhagen, Universitetsparken 5, 2100 Copenhagen $\varnothing$, Denmark e-mail: collamore@math.ku.dk

\section{A. Höing}

Department of Mathematics, Swiss Federal Institute of Technology, 8092 Zurich, Switzerland e-mail: ahoeing@math.ethz.ch 


\section{Introduction and summary}

In this article we consider Markov-modulated risk processes of the form

$$
S_{n}=\zeta_{1}+\cdots+\zeta_{n}
$$

where the increments $\left\{\zeta_{n}\right\}$ are driven by anderlying Markov chain $\left\{X_{n}\right\}$, as described explicitly in (2.1) below. We assume that $\left\{S_{n}\right\}$ has a positive mean drift, say $\mu$. Our objective is to study its large exceedance probabilities, more precisely,

$$
\mathbf{P}\left(\sup _{n \leq \delta u} S_{n}>u\right) \quad \text { as } u \rightarrow \infty .
$$

If $\delta<1 / \mu$, then the probability on the left of (1.2) tends to zero as $u \rightarrow \infty$; and since the time boundary at $\delta u$ grows as $u \rightarrow \infty$ and does so at a linear rate, this can be regarded as a large deviation problem. Our main result characterizes the rate of decay in (1.2) under the assumption, roughly, that $\hat{F}(u):=\mathbf{P}\left(\zeta_{1} \leq u \mid X_{1} \sim \pi\right)$ is heavytailed, where $\pi$ is the stationary distribution of $\left\{X_{n}\right\}$. In particular, $\hat{F}$ may belong to the regularly varying, lognormal, or Weibull classes of distribution functions.

The problem described in (1.2) is nonstandard and is motivated by certain aspects of risk management. As an important part of our study, we need to assume that the Markov chain takes values in a general state space, such as $\mathbb{R}^{d}$, which is uncountable. Thus our approach will depart significantly from classical risk theory, as described, e.g., in [1-3], where traditional ruin problems are studied for processes modulated by a Markov chain, but in a finite state space.

Our original motivation for (1.2) came from a problem in operational risk management, where there has been an interest in quantifying "repetitive" operational risk losses. This refers to capital loss incurred by a financial institution due to, say, documentation errors, system failure, or programming or information errors, which, following the Basel II accord, need to be estimated. The following stylized facts are well established: the individual losses are known to be heavy-tailed, consistent with regularly varying tails, while their arrival rates are closely correlated with traded volume in the market. It is natural to assume that the claims arrive at a Poisson rate, but that this arrival rate changes over time according to a volume index. With these considerations in mind, we are led to a model of the form

$$
S_{n}=\zeta_{1}+\cdots+\zeta_{n}, \quad \text { where } \zeta_{i}=\sum_{j=1}^{\mathfrak{N}_{i}} Z_{j}^{(i)}
$$

In this expression, $\mathfrak{N}_{n}$ is assumed to have a Poisson distribution with parameter $\lambda\left(X_{n}\right)$ for some function $\lambda$; and thus the total capital loss, $S_{n}$, is viewed as an aggregate of losses $\left\{Z_{j}^{(n)}\right\}$ which occur at a Poisson rate modulated by an external process $\left\{X_{n}\right\}$. Here, $\left\{X_{n}\right\}$ may be assumed to follow the $\mathrm{AR}(1)$ time series model, or an $\operatorname{ARMA}(p, q)$ model (based on the $p$-dimensional representation in [20], p. 28), or it may be governed by a far more general dependent process.

In this example, there is no premium payment, and the process $\left\{S_{n}\right\}$ has a positive drift, in the same direction as the claims process. It is of interest to characterize the 
chance that a large positive exceedance occurs over a time interval, namely (1.2), for a constant $\delta$ which is small compared with the natural ruin time for the process.

It is worth noting that models similar to (1.3) have also arisen in high frequency financial analysis (cf. [31, 32]). In this context, $Z_{j}^{(i)}$ in (1.3) denotes a discrete price jump, while, in the most realistic models, the intensity of the jumps is assumed to be time-dependent, modulated by an underlying Markov process. The asymptotic problem addressed in this paper can then be used to describe the probability that a large aggregate price movement occurs at some point within a time interval. Aside from its self-evident mathematical interest, this problem (and minor variants of it) also seems to be of some statistical relevance, for example, in change-point detection.

A further example - which we introduce to illustrate the breadth of our approachconsiders exceedances for the sums

$$
S_{n}=X_{1}+\cdots+X_{n}
$$

where

$$
X_{n}=A_{n} X_{n-1}+B_{n}, \quad n=1,2, \ldots, \quad X_{0}=x,
$$

and $\left\{\left(A_{n}, B_{n}\right): n=1,2, \ldots\right\}$ is an i.i.d. sequence of random variables. Then $\left\{X_{n}\right\}$ is a Markov chain on $\mathbb{R}$, and (1.5) is a so-called stochastic recurrence equation. The process $\left\{X_{n}\right\}$ plays a prominent role in the study of the $\operatorname{ARCH}(1)$ and $\operatorname{GARCH}(1,1)$ financial time series models (cf. $[13,21]$ ). The study of the sums $\left\{S_{n}\right\}$ in (1.4) has recently been initiated in [19], but under assumptions which are not satisfied by the $\operatorname{ARCH}(1)$ or $\operatorname{GARCH}(1,1)$ processes. It is of interest to consider the extremal behavior of (1.4) under conditions which will be satisfied by these models. In this context (1.4) could, in particular, describe the net price increase for a financial model where the jump sizes are, say, deterministic, but the number of jumps in a given time interval is governed by an $\mathrm{ARCH}(1)$ process. The generalization to random price jumps also seems feasible.

Motivated by these examples, our objective here is to consider-from a general theoretical viewpoint - the asymptotic behavior of (1.2), where $\left\{S_{n}\right\}$ is given as in (1.1) and is driven, we again emphasize, by a general state-space Markov chain, such as an $\operatorname{ARMA}(p, q)$ process or an $\operatorname{ARCH}(1)$ process, which will be typically assumed to be observable. (However, this last condition plays no role in our analysis, and an example to the contrary, where $\left\{X_{n}\right\}$ is latent, is given in Sect. 3.2 below.) Under some natural conditions, we shall show that

$$
\mathbf{P}\left(\sup _{n \leq \delta u} S_{n}>u\right) \sim C u \mathbf{P}(U>(1-\gamma) u) \quad \text { as } u \rightarrow \infty
$$

where $C$ and $\gamma$ are positive constants, and $U$ is a random variable describing the typical increase of $\left\{S_{n}\right\}$ over its regeneration cycles; see Lemma 2.1 below. Moreover, we shall show that, for both (1.3) and (1.5),

$$
\mathbf{P}(U>u) \sim D u \mathbf{P}_{\pi}\left(\zeta_{1}>u \mid X_{1} \sim \pi\right) \quad \text { as } u \rightarrow \infty,
$$

where $D$ is a positive constant (which, however, turns out to be different in two separate cases). 
We remark that results such as (1.7) appear to be of general interest, useful well beyond the context of this article. Moreover, it can be shown that there is a mathematical distinction in the Markovian behavior in (1.3) and (1.5), and this can be quantified.

Extensions of our work include applications to credit risk (a very preliminary result in this direction can be found in [18]) and statistical applications. (For a general discussion of large deviation asymptotics in statistics, see, e.g., [5].)

In the next section, we give a precise mathematical statement of (1.6) and, in Sect. 3, apply this result to the special cases (1.3) and (1.5). The proof of the main result (1.6) is then given in Sect. 4, while some technical results and their proofs can be found in the Appendix.

\section{Statement of results}

We now turn to a precise mathematical formulation of our problem. Let $\left\{X_{n}: n=0,1, \ldots\right\}$ be a Markov chain defined on a probability space $(\Omega, \mathfrak{F}, \mathbf{P})$, and assume that $\left\{X_{n}\right\}$ takes values in a state space $\mathbb{S}$ with $\sigma$-algebra $\mathfrak{S}$, which is assumed to be countably generated. Suppose that $\left\{X_{n}\right\}$ is time-homogeneous with onestep transition kernel $P(x, A)=: \mathbf{P}\left(X_{n+1} \in A \mid X_{n}=x\right)$ and $k$-step transition kernel $P^{k}=P^{k-1} P$ for $k>1$. Moreover, assume that $\left\{X_{n}\right\}$ is aperiodic and irreducible with respect to a maximal irreducibility measure $\varphi$. For the definitions and discussion of these conditions, see [20]. (In our examples, we always have $\mathbb{S}=\mathbb{R}^{d}$ equipped with the Borel $\sigma$-algebra, and $\varphi$ may be taken to be Lebesgue measure on $\mathbb{R}^{d}$.)

Next introduce a sequence of $\mathbb{R}$-valued random variables $\left\{\zeta_{n}: n=1,2, \ldots\right\}$, where

$$
\mathbf{P}\left(\left(X_{n+1}, \zeta_{n+1}\right) \in A \times \Gamma \mid \mathfrak{F}_{n}\right)=\mathbf{P}\left(\left(X_{n+1}, \zeta_{n+1}\right) \in A \times \Gamma \mid X_{n}\right)
$$

and

$$
\mathbf{P}\left(\left(X_{n+1}, \zeta_{n+1}\right) \in A \times \Gamma \mid X_{n}=x\right)=\int_{A} P(x, d y) \eta(y, \Gamma)
$$

for some family of probability measures $\{\eta(x, \Gamma): x \in \mathbb{S}, \Gamma \in \mathfrak{R}\}$, where $\mathfrak{R}$ denotes the Borel $\sigma$-algebra on $\mathbb{R}$, and $\mathfrak{F}_{n}=\sigma\left(X_{0}, \ldots, X_{n}, \zeta_{1}, \ldots, \zeta_{n}\right)$.

Our objective is to study the asymptotic behavior of the sums

$$
S_{n}:=\zeta_{1}+\cdots+\zeta_{n}
$$

in a setting where $\left\{S_{n}\right\}$ has a positive mean drift, namely,

$$
\mu:=\int_{\mathbb{S} \times \mathbb{R}} \pi(d y) s \eta(y, d s) \in(0, \infty),
$$

where $\pi$ denotes the stationary distribution of $\left\{X_{n}\right\}$, which is assumed to exist.

Minorization condition: There exist a $\varphi$-positive set $C \in \mathfrak{S}$, a probability measure $v$ on $(\mathbb{S}, \mathfrak{S})$, and positive constants $\delta>0$ and $k \in \mathbb{Z}_{+}$such that

$$
\delta \mathbf{1}_{C}(x) v(A) \leq P^{k}(x, A) \quad \text { for all } x \in \mathbb{S} \text { and } A \in \mathfrak{S} .
$$


The set $C$ appearing in $(\mathfrak{M})$ is called a "small set".

Condition $(\mathfrak{M})$ is an exceedingly weak requirement, which is roughly equivalent to "Harris recurrence" (see, e.g., [20]).

Under (M), an important result—first established in [4] and [28]—states that the Markov chain is regenerative.

Lemma 2.1 (Nummelin-Athreya-Ney) Assume (M). Then there exists a sequence of random times $0<T_{0}<T_{1}<\cdots$ such that:

(i) $\left\{T_{j+1}-T_{j}: j \in \mathbb{N}\right\}$ is a sequence of i.i.d. and a.s. finite random variables;

(ii) the random blocks $\left\{X_{T_{j}}, \ldots, X_{T_{j+1}-1}\right\}, j=0,1, \ldots$, are independent;

(iii) $\mathbf{P}\left(X_{T_{j}} \in A \mid \mathfrak{F}_{T_{j}-1}\right)=v(A)$ for all $A \in \mathfrak{S}$.

(Here, $\mathbb{N}=\{0,1, \ldots\}$ denotes the set of natural numbers.)

Let $\tau_{j}:=T_{j+1}-T_{j}, j \geq 0$, and $\tau_{0}:=T_{0}$ denote the inter-regeneration times. We shall generally need to assume that these times have finite exponential moments, and for this purpose we introduce the following:

Drift condition: There exist a small set $C \in \mathfrak{S}$, a function $V \geq 1(V \not \equiv \infty)$, and constants $\beta>0$ and $b<\infty$ such that

$$
\int_{\mathbb{S}} V(y) P(x, d y) \leq(1-\beta) V(x)+b \mathbf{1}_{C}(x) \text { for all } x \in \mathbb{S} .
$$

Next, we turn to some distributional assumptions on the process $\left\{S_{n}\right\}$. Given the regeneration times $0<T_{0}<T_{1}<\cdots$ of Lemma 2.1, set

$$
\begin{gathered}
U_{j}=\zeta_{T_{j-1}}+\cdots+\zeta_{T_{j}-1}, \quad j=0,1, \ldots\left(\text { where } T_{-1}=1\right) \\
\check{U}_{j}^{-}=\min \left\{0, \zeta_{T_{j-1}}\right\}+\cdots+\min \left\{0, \zeta_{T_{j}-1}\right\}, \quad j=0,1, \ldots
\end{gathered}
$$

Then $\left\{U_{j}: j=1,2, \ldots\right\}$ is an i.i.d. sequence of random variables, which is independent of $U_{0}$ (and similarly for $\left\{\check{U}_{j}^{-}: j=0,1, \ldots\right\}$ ). We shall frequently let $U \stackrel{d}{=} U_{j}, j \geq 1$, and $\check{U}^{-} \stackrel{d}{=} \check{U}_{j}^{-}, j \geq 1$, denote random variables describing typical increments of these processes.

The original risk process (2.2) can then be written as

$$
S_{n}=U_{0}+U_{1}+\cdots+U_{N^{*}-1}+\left(\zeta_{T_{N^{*}-1}}+\cdots+\zeta_{n}\right)
$$

where $N^{*}:=\inf \left\{j \geq 0: T_{j}>n\right\}(\inf \emptyset:=\infty)$. The first and last terms turn out to be asymptotically negligible, and so it makes sense to focus on the distribution function $F$ of a typical increment $U$, which-based on our motivating examples-will mainly be assumed to be regularly varying.

Recall that a distribution $G$ is said to be regularly varying with index $\alpha$ (written $G \in \mathcal{R}(\alpha))$ if its tail distribution $\bar{G}(u):=1-G(u)$ satisfies

$$
\bar{G}(u) \sim L(u) u^{-\alpha} \quad \text { as } u \rightarrow \infty,
$$


where $L$ is a slowly varying function, i.e., $\lim _{u \rightarrow \infty} L(t u) / L(u)=1$ for all $t>0$. The distribution $G$ is said to have a lognormal-type tail with parameter $\gamma$ (written $G \in \mathrm{LN}(\gamma))$ if

$$
\bar{G}(u) \sim c u^{\beta} e^{-\lambda \log ^{\gamma} u} \quad \text { as } u \rightarrow \infty
$$

for some $\beta \in \mathbb{R}, \gamma>1$, and $c>0 . G$ is said to have a Weibull-type tail with parameter $\alpha$ (written $G \in \mathrm{WE}(\alpha))$ if

$$
\bar{G}(u) \sim c u^{\beta} e^{-\lambda u^{\alpha}} \quad \text { as } u \rightarrow \infty
$$

for some $\beta \in \mathbb{R}, \alpha \in(0,1), \lambda>0$, and $c>0$. For a more detailed description of these distributions, see [13].

Theorem 2.2 Suppose that $\left\{X_{n}\right\}$ is a Markov chain satisfying (M) and (D) and assume that $\mathbf{P}\left(\check{U}^{-}<-u\right)=o(\mathbf{P}(U>u))$ as $u \rightarrow \infty$.

(i) Suppose that either: (a) $F \in \mathcal{R}(\alpha)$ for some $\alpha>1$; or (b) $F \in \mathrm{LN}(\gamma)$; or (c) $F \in \mathrm{WE}(\alpha)$ for some $\alpha \in(0,1 / 3)$. Then

$$
\psi_{\delta, x}(u):=\mathbf{P}\left(\sup _{n \leq \delta u} S_{n}>u \mid X_{0}=x\right) \sim \frac{\delta u}{\mathbf{E}[\tau]} \bar{F}((1-\delta \mu) u)
$$

as $u \rightarrow \infty$ for any positive constant $\delta<1 / \mu$ and $\varphi$-a.a. $x$.

(ii) If $F \in \mathrm{WE}(\alpha)$ for $\alpha \in[1 / 3,1 / 2)$, then, for any $\theta \in(1-\alpha, 2-3 \alpha)$,

$$
\psi_{\delta, x}^{(\theta)}(u):=\mathbf{P}\left(\sup _{n \leq \delta u^{\theta}} S_{n}>u \mid X_{0}=x\right) \sim \frac{\delta u^{\theta}}{\mathbf{E}[\tau]} \bar{F}\left(u-\delta \mu u^{\theta}\right)
$$

as $u \rightarrow \infty$ for any positive constant $\delta$ and $\varphi$-a.a. $x$.

The proof of Theorem 2.2 will be given below in Sect. 4 .

Remark 2.3 In the case $F \in \mathcal{R}(\alpha)$ with $\alpha>1$, (2.6) reduces to

$$
\psi_{\delta, x}(u) \sim \frac{\delta u}{\mathbf{E}[\tau]}(1-\delta \mu)^{-\alpha} \bar{F}(u) \quad \text { as } u \rightarrow \infty .
$$

\section{Applications}

We now return to the motivating examples introduced in Sect. 1. Before proceeding, we should like to stress that the applications and models presented below should be viewed as examples of how Markov modulation can occur. In the case of operational risk, in particular, it is not our objective to strive for a full model for this kind of risk. 


\subsection{Repetitive operational risk losses}

Formally, operational risk has been defined in [6] as "the risk of losses resulting from inadequate or failed internal processes, people and systems, or from external events." Our focus is on "repetitive" operational risk, which refers, more specifically, to systematic errors arising sporadically over time and caused, e.g., by system failure, programming or information errors, or other back-office errors, such as transaction risk or control risk; see [9].

Some stylized features of these losses are: (i) the sizes of the individual losses, or "claims," appear to be independent and quite heavy-tailed, consistent with Pareto tails; and (ii) their occurrences are irregularly-spaced in time and appear in clusters, which tend to coincide with large bursts of traded volume in the market. See [12] for a more detailed discussion. In particular, it is reasonable to assume that the losses occur at a Poisson rate, as in the classical ruin problem, but that the intensity of losses depends on an external process, $\left\{X_{n}\right\}$, which is observed. This suggests a model of the form

$$
S_{n}=\zeta_{1}+\cdots+\zeta_{n}, \quad \text { where } \zeta_{i}=\sum_{j=1}^{\mathfrak{N}_{i}} Z_{j}^{(i)}
$$

Here, $\left\{Z_{j}^{(n)}: n \in \mathbb{Z}_{+}, j \in \mathbb{Z}_{+}\right\}$is an i.i.d. sequence of random variables with common distribution function $F_{Z} \in \mathcal{R}(\alpha)$ or, more generally, $F_{Z} \in \mathcal{S}$, where $\mathcal{S}$ denotes the subexponential class (as defined in [13]). For any fixed $n, \mathfrak{N}_{n}$ is assumed to have a Poisson distribution with parameter $\lambda\left(X_{n}\right)$ for some function $\lambda: \mathbb{S} \rightarrow[0, \infty)$. Moreover, it is assumed that, conditioned on a realization $\left\{x_{n}\right\}$ of $\left\{X_{n}\right\}$, the random variables $\mathfrak{N}_{1}, \mathfrak{N}_{2}, \ldots$ are independent, and $\left\{Z_{j}^{(n)}\right\}$ is independent of $\left\{\mathfrak{N}_{n}\right\}$. We regard $\left\{X_{n}\right\}$ as a volume index, which we assume to be a Markov chain on $\mathbb{R}^{d}$ (say).

The simplest choice for the intensity would be to take $\lambda(x) \in\left\{\lambda_{1}, \lambda_{2}\right\}$, which would correspond to a regime-switching model, as has been introduced, e.g., in [16] and [17]. Another reasonable choice would be to take $\lambda(x)=K x, x \geq c$, and $\lambda(x)=0$ otherwise for some threshold $c \geq 0$ and constant $K>0$.

Applying Theorem 2.2 yields (2.8), but this result is useless without a characterization of $\bar{F}$, namely the distribution of $U$, which describes the increase of $\left\{S_{n}\right\}$ over its regeneration cycles.

To this end, let

$$
\begin{aligned}
Y_{n} & =\lambda\left(X_{1}\right)+\cdots+\lambda\left(X_{n}\right) ; \\
\Theta_{Y}(\xi) & =\limsup _{n \rightarrow \infty} \frac{1}{n} \log \mathbf{E}\left[e^{\xi Y_{n}}\right] .
\end{aligned}
$$

Then $\Theta_{Y}$ is the "Gärtner-Ellis limit" from large deviation theory, cf. [11], Sect. 2.3; this is closely related to the spectral radius of an associated transform kernel, cf., e.g., [10].

Proposition 3.1 Assume the model described in (3.1), where $\left\{X_{n}\right\}$ is a Markov chain satisfying $(\mathfrak{M})$ and $(\mathfrak{D})$ and suppose that $\hat{\mu}:=\mathbf{E}_{\pi}[\lambda(X)]<\infty$. 
(i) If $F_{Z} \in \mathcal{R}(\alpha)$ with $\alpha>0$, and $\mathbf{E}_{\pi}\left[\mathfrak{N}^{\beta}\right]<\infty$ for some $\beta>\alpha$, then

$$
\bar{F}(u) \sim \hat{\mu} \mathbf{E}[\tau] \bar{F}_{Z}(u) \quad \text { as } u \rightarrow \infty .
$$

(ii) If $F_{Z} \in \mathcal{S}$ and $\Theta_{Y}(\xi)<\infty$ for some $\xi>0$, then (3.2) holds.

(In the statement of the proposition, $\mathbf{E}_{\pi}[\cdot]$ denotes the expected value under the assumption that the underlying Markov chain assumes its stationary distribution.)

The proof of Proposition 3.1 will be given below in the Appendix.

Remark 3.2 Our requirement in (ii) that $\Theta_{Y}(\xi)<\infty$ for some $\xi>0$-which is stronger than the moment assumption in (i) — can be seen to hold rather generally. For example, if $\left\{X_{n}\right\}$ is an $\operatorname{ARMA}(p, q)$ process with Gaussian errors and $\lambda(x)=\max \{x, c\}$ for some $c \geq 0$, then $\Theta_{Y}$ is finite everywhere. Moreover, if $\lambda$ is bounded, then $\Theta_{Y}$ is finite everywhere for any process $\left\{X_{n}\right\}$.

Combining Theorem 2.2 (as given in (2.8)) with Proposition 3.1 yields

$$
\psi_{\delta, x}(u) \sim \hat{\mu} \delta u(1-\delta \mu)^{-\alpha} \bar{F}_{Z}(u) \quad \text { as } u \rightarrow \infty .
$$

To analyze the validity of (3.3) statistically, we should need data for both the claim sizes and traded volume. The first is not publicly available, although internal data we have seen suggests that our model is reasonable. However, data for traded volume can be easily obtained. For example, an analysis of the weekly volume of Nokia quotes for the period of January 2000-December 2003 suggests that the Nokia time series follows an $\mathrm{AR}(1)$ process, whereas the same analysis for the corresponding Intel data suggests that it follows an $\mathrm{AR}(2)$ process. Both are examples of a general state-space Markov chain satisfying conditions $(\mathfrak{M})$ and $(\mathfrak{D})$ of Sect. 2.1. (Note that, in the $\operatorname{AR}(2)$ case, the state space of the Markov chain is actually $\mathbb{R}^{2}$, based on the representation in [20], p. 28.)

Focusing on the Nokia data set, a statistical analysis suggests that $X_{n}=a X_{n-1}+$ $\varepsilon_{n}$, where $\left\{\varepsilon_{n}\right\} \sim \operatorname{Normal}\left(0, \sigma^{2}\right)$ for $a=0.90$ and $\sigma=5.66 \times 10^{6}$. Suppose, moreover, that $\left\{Z_{j}^{(n)}\right\}$ has a Pareto(3/2,3) distribution and that the intensity function $\lambda$ has the form $\lambda(x)=x, x \geq c=1.9 \times 10^{7}$, and $\lambda(x)=0$ otherwise. We then obtain the simulated losses $\left\{\zeta_{n}\right\}$ displayed in Fig. 1. These simulated losses closely resemble
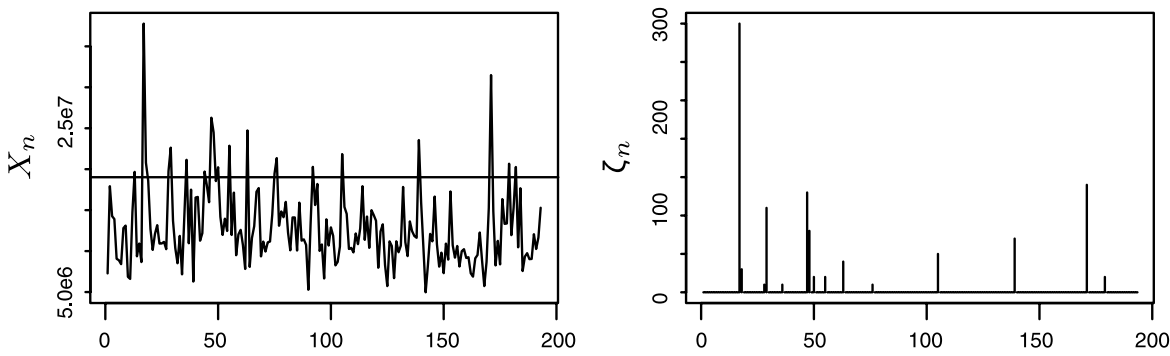

Fig. 1 Nokia volume (left) and corresponding loss process (right) 
what is seen in practice: the individual losses have periods of low and high intensity, with changes in the intensity driven by large changes in the volume process.

It is interesting to note that (3.1) could also apply as a model for high frequency financial data. In a financial market, one might assume that discrete jumps occur when quotes are posted and that the quotes arise at a Poisson rate, governed by a Poisson process which is inhomogeneous and modulated by an underlying Markov process. If the Markov process is ergodic, then estimates for means and variances have recently been given in [31]. Our results deal with extremal behavior, but our assumptions on the underlying process, $\left\{X_{n}\right\}$, are otherwise roughly similar to theirs.

\subsection{GARCH models and stochastic recurrence equations}

The popular $\operatorname{GARCH}(1,1)$ financial time series model of [8] assumes that the logarithmic returns of a stock, $\left\{Y_{n}\right\}$, satisfy

$$
Y_{n}=\sigma_{n} Z_{n}, \quad \text { where } \sigma_{n}^{2}=a_{0}+b_{1} \sigma_{n-1}^{2}+a_{1} Y_{n-1}^{2},
$$

and $\left\{Z_{n}\right\}$ is an i.i.d. sequence of $\operatorname{Normal}(0,1)$ random variables.

The intuition behind (3.4) is that, in practice, observed volatilities exhibit correlation with respect to the absolute values of the returns, but little correlation with respect to the actual returns. See [21] for a survey on GARCH models and their properties.

The mathematical analysis of the $\operatorname{GARCH}(1,1)$ process can be based on the fact that it satisfies a stochastic recurrence equation. Namely, if $X_{n}=\sigma_{n}^{2}$, then

$$
X_{n}=A_{n} X_{n-1}+B_{n},
$$

where

$$
A_{n}=b_{1}+a_{1} Z_{n-1}^{2} \text { and } B_{n}=a_{0} .
$$

If we now let $\left\{\left(A_{n}, B_{n}\right)\right\}$ be an arbitrary i.i.d. sequence of random variables and let $\left\{X_{n}\right\}$ be a sequence of random variables satisfying (3.5), $n=1,2, \ldots$ (where $X_{0}=$ $x \in \mathbb{R})$, then it is of mathematical interest and potential statistical relevance to study the extremal behavior of the sums

$$
S_{n}=X_{1}+\cdots+X_{n}
$$

This setting agrees with our general assumptions, since the process $\left\{X_{n}\right\}$ is a Markov chain on $\mathbb{R}$, which is known to satisfy the regularity conditions $(\mathfrak{M})$ and $(\mathfrak{D})$ (for the latter, see [7]).

Thus we may apply Theorem 2.2 as before, but once again, a characterization of $\bar{F}$ is needed for this result to be useful. Let

$$
\Lambda_{A}(\xi)=\log \mathbf{E}\left[A^{\xi}\right], \quad \Lambda_{B}(\xi)=\log \mathbf{E}\left[B^{\xi}\right] \quad \text { for all } \xi \in \mathbb{R} .
$$

Proposition 3.3 Assume that $\kappa:=\sup \left\{\xi: \Lambda_{A}(\xi) \leq 0\right\} \in(0, \infty)$ and that the functions $\Lambda_{A}$ and $\Lambda_{B}$ are finite in a neighborhood of $\kappa$. Then, for some constant $C \in(0, \infty)$,

$$
\bar{F}(u) \sim C u^{-\kappa} \quad \text { as } u \rightarrow \infty .
$$


The proof is given in the Appendix.

By Theorem 2.2 and Proposition 3.3,

$$
\psi_{\delta, x}(u) \sim C u((1-\delta \mu) u)^{-\kappa} \quad \text { as } u \rightarrow \infty .
$$

As an interesting mathematical observation, we note that (3.7) is structurally different from (3.3); that is to say, both (3.3) and (3.7) can be put in the general form

$$
\psi_{\delta, x}(u) \sim D u \mathbf{P}\left(\zeta_{1}>u \mid X_{1} \sim \pi\right) \quad \text { as } u \rightarrow \infty,
$$

where $\pi$ is the stationary distribution of $\left\{X_{n}\right\}$, but the constant $D$ will be different in the two separate cases. Hence it is not possible to obtain a general result which describes the behavior over the regeneration cycles (as in Propositions 3.1 and 3.3) which holds in full generality. Indeed, from a mathematical perspective, an exceedance in Proposition 3.1 results from a single large jump over a given regeneration cycle, while an exceedance in Proposition 3.3 results from a large accumulation due to atypical path behavior of the sequence $\left\{A_{n}\right\}$ over the cycle. In this second case, the length of the cycle leading to ruin will be unusually large, while the individual increments leading to ruin will be "twisted" in the sense of large deviation theory. Thus, in this case, no individual term of the sequence $\left\{A_{n}\right\}$ will have an unusually large value, but rather an unusual combination of random variables will lead to the exceedance described in (3.6).

There has been very little work dealing with the sums of processes governed by stochastic recurrence equations. The notion was recently introduced in [19] but, in their case, the sequence $\left\{B_{n}\right\}$ is assumed to have heavier tails than $u^{-\kappa}$; i.e., their setting does not agree with that of the standard $\operatorname{GARCH}(1,1)$ process and leads to different tail asymptotics from those obtained here.

\section{Proof of Theorem 2.2}

Case $1 F \in \mathcal{R}(\alpha)$ for some $\alpha>1$.

Upper bound. We begin by considering the probability that the process is in the state of ruin at some regeneration time. To this end, let $\left\{U_{j}: j=0,1, \ldots\right\}$ denote the increase in $\left\{S_{n}\right\}$ over its regeneration cycles, as defined formally in (2.4). Then the probability that ruin is incurred at a time of regeneration is

$$
\mathbf{P}\left(U_{0}+\cdots+U_{j}>u \text { for some } j<N(u)\right)
$$

where

$$
N(u)=\inf \left\{j: T_{j}>\delta u\right\}
$$

and where $\left\{T_{j}\right\}$ are the regeneration times of Lemma 2.1. Thus, $N(u)-1$ denotes the number of regenerations which occur up to time $\delta u$, and (4.1) describes the probability that ruin is incurred at one of these times. 
To determine (4.1), we first introduce a standard ladder height construction; namely, let

$$
\begin{aligned}
Y_{n} & =U_{0}+\cdots+U_{n}, \quad n=0,1, \ldots ; \\
T_{0}^{+} & =\inf \left\{n: Y_{n}>0\right\} ; \quad T_{j+1}^{+}=\inf \left\{n>T_{j}^{+}: Y_{n}>Y_{T_{j}^{+}}\right\}, \quad j=0,1, \ldots ; \\
\tau_{0}^{+} & =T_{0}^{+} ; \quad \tau_{j+1}^{+}=T_{j+1}^{+}-T_{j}^{+}, \quad j=0,1, \ldots ; \\
L_{0} & =Y_{T_{0}^{+}} ; \quad L_{j+1}=Y_{T_{j+1}^{+}}-Y_{T_{j}^{+}}, \quad j=0,1, \ldots
\end{aligned}
$$

We are primarily interested in the ladder heights $\left\{L_{j}\right\}$, which describe the points of increase of the process $\left\{Y_{n}\right\}$ just defined. Note that since $\left\{U_{n}\right\}_{n \geq 1}$ is i.i.d. and independent of $U_{0}$, it follows that $\left\{L_{j}\right\}_{j \geq 1}$ and $\left\{\tau_{j}^{+}\right\}_{j \geq 1}$ likewise are i.i.d. and independent of $L_{0}$ and $\tau_{0}^{+}$, respectively.

Going back to (4.1) and observing that the sequence $\left\{L_{0}+\cdots+L_{j}\right\}$ is increasing, we obtain

$$
\mathbf{P}\left(U_{0}+\cdots+U_{j}>u, \text { for some } j<N(u)\right)=\mathbf{P}\left(L_{0}+\cdots+L_{M(u)-1}>u\right),
$$

where

$$
M(u)=\inf \left\{j: T_{j}^{+} \geq N(u)\right\},
$$

i.e., $M(u)-1$ is the number of ladder heights which occur by time $\delta u$.

Now by Wald's identity the expected number of steps until the occurrence of a typical ladder height time is $\mathbf{E}[\tau] \mathbf{E}\left[\tau^{+}\right]$. Hence, large deviation estimates for renewal processes (as given in Lemma 5.2 below) yield

$$
\mathbf{P}\left(M(u)-\frac{\delta u}{\mathbf{E}[\tau] \mathbf{E}\left[\tau^{+}\right]}>\epsilon u\right) \stackrel{\exp }{\rightarrow} 0 \quad \text { as } u \rightarrow \infty
$$

for any $\epsilon>0$. Thus, if we define

$$
m(u)=\left\lfloor\frac{\delta u}{\mathbf{E}[\tau] \mathbf{E}\left[\tau^{+}\right]}+\epsilon u\right\rfloor \quad \text { and } \quad \mathcal{M}_{u}=\{M(u) \leq m(u)\}
$$

then

$$
\mathbf{P}\left(L_{0}+\cdots+L_{M(u)-1}>u\right) \leq \mathbf{P}\left(L_{0}+\cdots+L_{m(u)}>u\right)+\mathbf{P}\left(\mathcal{M}_{u}^{c}\right),
$$

where $\mathbf{P}\left(\mathcal{M}_{u}^{c}\right) \stackrel{\exp }{\rightarrow} 0$.

We now focus on the first term on the right of (4.7). A result of [25] states that if $\left\{V_{n}\right\}$ is an i.i.d. sequence with mean zero and distribution $G \in \mathcal{R}(\alpha)$, where $\alpha>1$, then

$$
\mathbf{P}\left(V_{1}+\cdots+V_{n}>s\right) \sim n \bar{G}(s) \quad \text { as } n \rightarrow \infty
$$

for all $s \geq c n$, where $c$ is an arbitrary constant. 
To apply this result in our setting, we first need to characterize the mean of $L$ and its distribution function, where $L \stackrel{d}{=} L_{j}, j \geq 1$, is a typical ladder height. By Lemma 5.2 of [27] we have $\mu=\mathbf{E}[U] / \mathbf{E}[\tau]$. Then, by Wald's identity,

$$
\mathbf{E}[L]=\mathbf{E}\left[\tau^{+}\right] \cdot \mu \mathbf{E}[\tau]
$$

Moreover, for $\left\{Y_{n}\right\}$ given as above but with $U_{0}=0$,

$$
\begin{aligned}
\mathbf{P}(L>u) & =\sum_{n=1}^{\infty} \mathbf{P}\left(Y_{n}>u \text { and } Y_{k} \leq 0, \forall k<n\right) \\
& \leq \sum_{n=1}^{\infty} \mathbf{P}\left(\tau^{+}>n-1\right) \mathbf{P}\left(U_{n}>u\right)=\mathbf{E}\left[\tau^{+}\right] \bar{F}(u)
\end{aligned}
$$

Conversely, if $R_{-}(E):=\mathbf{E}\left[\sum_{n=1}^{\tau^{+}-1} \mathbf{1}_{\left\{Y_{n} \in E\right\}}\right]$ denotes the pre- $\tau^{+}$occupation measure, then, for any given $\Delta>0$,

$$
R_{-}([-a, 0]) \geq \mathbf{E}\left[\tau^{+}\right]-\Delta \text { for sufficiently large } a,
$$

since $\tau^{+}$is finite a.s. Consequently,

$$
\mathbf{P}(L>u) \geq \int_{-a}^{0} \bar{F}(u-v) R_{-}(d v) \sim\left(\mathbf{E}\left[\tau^{+}\right]-\Delta\right) \bar{F}(u)
$$

by Proposition IX.1.5 of [1]. From (4.10) and (4.12) we conclude that $L \in \mathcal{R}(\alpha)$. Finally, note that, by Lemma 5.1 of the Appendix, when $L$ is replaced with $L_{0},(4.9)$ and (4.10) remain valid up to multiplication by a finite constant. Hence, from (4.8) we now conclude that

$$
\begin{aligned}
\mathbf{P}\left(L_{1}+\cdots+L_{m(u)}>u\right) & \sim m(u) \mathbf{P}(L>u-m(u) \mathbf{E}[L]) \\
& \sim\left(\frac{\delta u}{\mathbf{E}[\tau]}+\epsilon u \mathbf{E}\left[\tau^{+}\right]\right) \mathbf{P}\left(U>(1-\delta \mu) u-\epsilon^{\prime} u\right),
\end{aligned}
$$

where $\epsilon^{\prime}=\epsilon \mu \mathbf{E}[\tau] \mathbf{E}\left[\tau^{+}\right]$. Moreover, since $L_{0}$ has an upper bound for its tail distribution which - up to multiplication by a constant—is equivalent to that of $L$, it follows that (4.13) holds with $L_{0}+\cdots+L_{m(u)}$ in place of $L_{1}+\cdots+L_{m(u)}$.

Substituting (4.13) into (4.3) and (4.7) and recalling that $\mathbf{P}\left(\mathcal{M}_{u}^{c}\right) \stackrel{\exp }{\longrightarrow} 0$, by Lemma 5.2, we obtain

$$
\begin{gathered}
\limsup _{u \rightarrow \infty} \frac{\mathbf{P}\left(U_{1}+\cdots+U_{j}>u, \text { for some } j<N(u)\right)}{(\delta u / \mathbf{E}[\tau]) \bar{F}((1-\delta \mu) u)} \\
\quad \leq \limsup _{\epsilon^{\prime} \rightarrow 0} \limsup _{u \rightarrow \infty} \frac{\mathbf{P}\left(U>(1-\delta \mu) u-\epsilon^{\prime} u\right)}{\mathbf{P}(U>(1-\delta \mu) u)}=1,
\end{gathered}
$$

where the last step follows from the fact that $F \in \mathcal{R}(\alpha)$, where $F$ is the distribution function of $U$. 
Thus we have characterized the probability that the process is in the state of ruin at a regeneration time, and our final objective is to determine the probability of ruin for the original sums $\left\{S_{n}\right\}$. To this end we employ a truncation argument as follows. Let $\zeta_{n}^{(K)}=\max \left\{\zeta_{n},-K\right\}$,

$$
S_{n}^{(K)}=\zeta_{1}^{(K)}+\cdots+\zeta_{n}^{(K)}
$$

and let $U_{j}^{(K)}$, etc., be defined in the obvious way, with $\left\{\zeta_{n}^{(K)}\right\}$ in place of $\left\{\zeta_{n}\right\}$.

Note that $\zeta_{n}^{(K)} \geq \zeta_{n}$ and hence $S_{n}^{(K)} \geq S_{n}$ for all $n$. Thus,

$$
\psi_{\delta, x}(u) \leq \mathbf{P}\left(S_{n}^{(K)}>u, \text { for some } n \leq \delta u\right) .
$$

Set $\mathcal{L}_{u}=\left\{\tau_{j} \leq u / K^{2}\right.$, for all $\left.j<N(u)\right\}$. Then fix $n$ and, looking forward in time, compare the magnitude of $\left\{S_{n}^{(K)}\right\}$ at time $n$ with that at $T^{*}:=$ the next regeneration time. Note that $S_{n}^{(K)}-S_{T^{*}}^{(K)} \leq u / K$ on $\mathcal{L}_{u}$. This is because the length of any given regeneration cycle is not larger than $u / K^{2}$, and the negative increments of the process, under truncation, are not larger than $K$. Consequently,

$$
\psi_{\delta, x}(u) \leq \mathbf{P}\left(U_{1}^{(K)}+\cdots+U_{j}^{(K)}>\left(1-\frac{1}{K}\right) u \text {, for some } j<N(u)\right)+\mathbf{P}\left(\mathcal{L}_{u}^{c}\right) .
$$

Next observe that $\lim _{K \rightarrow \infty} \mathbf{E}\left[U^{(K)}\right] / \mathbf{E}[U]=1$, while

$$
\mathbf{P}\left(U^{(K)}>u\right) \leq \mathbf{P}\left(U-\check{U}^{-}>u\right) \sim \mathbf{P}(U>u) \quad \text { as } u \rightarrow \infty,
$$

since by assumption $\mathbf{P}\left(\check{U}^{-}<-u\right)=o(\mathbf{P}(U>u))$. Therefore the proof leading to (4.14) may be repeated to obtain

$$
\limsup _{K \rightarrow \infty} \limsup _{u \rightarrow \infty} \frac{\mathbf{P}\left(U_{1}^{(K)}+\cdots+U_{j}^{(K)}>(1-1 / K) u, \text { for some } j<N(u)\right)}{(\delta u / \mathbf{E}[\tau]) \bar{F}((1-\delta \mu) u)} \leq 1,
$$

where we have used that $F \in \mathcal{R}(\alpha)$. By (4.15) the upper bound is now established, provided that $\mathbf{P}\left(\mathcal{L}_{u}^{c}\right) \rightarrow 0$ at a rate faster than $u \bar{F}((1-\delta \mu) u)$.

For this purpose, note that, by condition (D) and Theorem 15.0.1 of [20],

$$
\mathbf{E}\left[e^{\xi\left(T_{j+1}-T_{j}\right)}\right]<\infty \quad \text { for some } \xi>0
$$

for all $j \geq 0$. (To be entirely precise, Meyn and Tweedie consider a single visit to the set $C$ in $(\mathfrak{M})$ prior to regeneration, whereas it may actually be necessary to consider multiple visits to $C$. However, this added complication can be handled, e.g., based on the characterization in [26], p. 7.) Moreover, by Chebyshev's inequality,

$$
\mathbf{P}\left(\mathcal{L}_{u, j+1}^{c}\right):=\mathbf{P}\left(T_{j+1}-T_{j}>\frac{u}{K^{2}}\right) \leq e^{-\xi u / K^{2}} \mathbf{E}\left[e^{\xi\left(T_{j+1}-T_{j}\right)}\right]
$$

for all $j \geq 0$ (and the case of $\mathcal{L}_{u, 0}$ can be handled similarly by using Lemma 5.1 below). Consequently,

$$
\mathbf{P}\left(\mathcal{L}_{u}^{c}\right) \leq \text { const } \cdot \delta u e^{-\xi u / K^{2}} \quad \text { for some } \xi>0 .
$$


Lower bound. For any $\epsilon>0$, let

$$
k(u)=\left\lfloor\frac{\delta u}{\mathbf{E}[\tau]}-\epsilon u\right\rfloor \quad \text { and } \quad \mathcal{K}_{u}=\{N(u) \geq k(u)\},
$$

where $N(u)-1$ again denotes the number of regenerations which occur by time $\delta u$. On the set $\mathcal{K}_{u}$, there are at least $k(u)-1$ regenerations by time $\delta u$, and so $T_{k(u)-1} \leq \delta u$. Hence

$$
\psi_{\delta}(u):=\mathbf{P}\left(S_{n}>u \text {, for some } n \leq \delta u\right) \geq \mathbf{P}\left(U_{k(u)-1}>u\right)-\mathbf{P}\left(\mathcal{K}_{u}^{c}\right) .
$$

Since $\mathbf{E}[U]=\mu \mathbf{E}[\tau]$, it follows by [25] (cf. (4.8) above) that

$$
\begin{aligned}
\mathbf{P}\left(U_{0}+\cdots+U_{k(u)-1}>u\right) & \sim k(u) \mathbf{P}(U>u-k(u) \mathbf{E}[U]) \\
& \sim\left(\frac{\delta u}{\mathbf{E}[\tau]}-\epsilon u\right) \mathbf{P}\left(U>(1-\delta \mu) u+\epsilon^{\prime} u\right),
\end{aligned}
$$

where $\epsilon^{\prime}=\epsilon \mu \mathbf{E}[\tau]$. (Once again, the initial term, $U_{0}$, can be handled by using Lemma 5.1 below.) Since $\mathbf{P}\left(\mathcal{K}_{u}^{c}\right) \stackrel{\exp }{\rightarrow} 0$, by Lemma 5.2, from (4.22) and (4.23) it follows that

$$
\begin{aligned}
& \liminf _{u \rightarrow \infty} \frac{\psi_{\delta}(u)}{(\delta u / \mathbf{E}[\tau]) \bar{F}((1-\delta \mu) u)} \\
& \geq \liminf _{\epsilon^{\prime} \rightarrow 0} \liminf _{u \rightarrow \infty} \frac{\mathbf{P}\left(U>(1-\delta \mu) u+\epsilon^{\prime} u\right)}{\mathbf{P}(U>(1-\delta \mu) u)}=1,
\end{aligned}
$$

where the last equality holds since $U$ has a regularly varying distribution function.

Case $2 F \in \mathrm{LN}(\gamma)$ or $F \in \mathrm{WE}(\alpha), \alpha \in(0,1 / 2)$.

We adopt the same notation as in Case 1 but, in contrast to (4.6), set

$$
\check{m}(u)=\left\lfloor\frac{\delta u}{\mathbf{E}[\tau] \mathbf{E}\left[\tau^{+}\right]}+\epsilon \check{a}((1-\delta \mu) u)\right\rfloor \quad \text { and } \quad \check{\mathcal{M}}_{u}=\{M(u) \leq \check{m}(u)\},
$$

where $\check{a}$ is the "auxiliary function" defined by

$$
\check{a}(u)= \begin{cases}u \log ^{1-\gamma} u / \lambda \gamma & \text { if } F \in \operatorname{LN}(\gamma), \\ u^{1-\alpha} / \lambda \alpha & \text { if } F \in \operatorname{WE}(\alpha) .\end{cases}
$$

First, we focus on the cases $F \in \mathrm{LN}(\gamma)$ and $F \in \mathrm{WE}(\alpha), \alpha \in(0,1 / 3)$.

Reasoning as before (cf. (4.3), (4.7)), we obtain

$$
\begin{aligned}
& \mathbf{P}\left(U_{0}+\cdots+U_{j}>u, \text { for some } j<\check{N}(u)\right) \\
& \quad \leq \mathbf{P}\left(L_{0}+\cdots+L_{\check{m}(u)}>u\right)+\mathbf{P}\left(\check{\mathcal{M}}_{u}^{c}\right) .
\end{aligned}
$$


Table 1 Threshold $d_{n}$ for different heavy-tailed distributions

\begin{tabular}{lll}
\hline $\operatorname{LN}(\gamma), \gamma \in(1,2]$ & $\operatorname{LN}(\gamma), \gamma>2$ & $\mathrm{WE}(\alpha)$ \\
\hline$n^{1 / 2} \log ^{1 / 2} n$ & $n^{1 / 2} \log ^{\gamma / 2} n$ & $n^{1 / 2(1-\alpha)}$ \\
\hline
\end{tabular}

Now if $\left\{V_{n}\right\}$ is an i.i.d. sequence of random variables with mean zero and distribution $G$ having a left tail which is, say, bounded, then a result of [23, 24] (cf. [22]) states that, for $G \in \mathrm{LN}(\gamma)$ or $G \in \mathrm{WE}(\alpha)$,

$$
\mathbf{P}\left(V_{1}+\cdots+V_{n}>s\right) \sim n \bar{G}(s) \quad \text { as } n \rightarrow \infty
$$

for any $s \in\left(d_{n} g_{n}, \infty\right)$, where $g_{n}$ is an arbitrary sequence such that $g_{n} \rightarrow \infty$ as $n \rightarrow \infty$ and $d_{n}$ is given as in Table 1 . In particular, we may take $s \geq c n$ for the cases $\operatorname{LN}(\gamma)$ and $\operatorname{WE}(\alpha), \alpha \in(0,1 / 2)$. Then (cf. (4.13))

$$
\begin{aligned}
\mathbf{P}\left(L_{1}+\cdots+L_{\check{m}(u)}>u\right) \sim \check{m}(u) \mathbf{P}(L>u-\check{m}(u) \mathbf{E}[L]) \\
\sim\left(\frac{\delta u}{\mathbf{E}[\tau]}+\epsilon \check{a}(u) \mathbf{E}\left[\tau^{+}\right]\right) \mathbf{P}\left(U>(1-\delta \mu) u-\epsilon^{\prime} \check{a}(u)\right),
\end{aligned}
$$

where $\epsilon^{\prime}=\mu \mathbf{E}[\tau] \mathbf{E}\left[\tau^{+}\right]$.

To establish an upper bound over the regeneration cycles (the equivalent of (4.14)), we need to show that

$$
\limsup _{\epsilon^{\prime} \rightarrow 0} \limsup _{u \rightarrow \infty} \frac{\mathbf{P}\left(U>(1-\delta \mu) u-\epsilon^{\prime} \check{a}((1-\delta \mu) u)\right)}{\mathbf{P}(U>(1-\delta \mu) u)} \leq 1 .
$$

To this end, note that, for the above choice of $\check{a}$,

$$
\lim _{v \rightarrow \infty} \mathbf{P}(U>v-\check{a}(v) Z \mid U>v) \rightarrow \mathbf{P}(Z>z)
$$

for some random variable $Z$ which has a continuous density on $(0, \infty)$; see, e.g., [22], p. 90. Hence (4.29) follows.

It remains to show that $\mathbf{P}\left(\hat{\mathcal{M}}_{u}^{c}\right)=o(\bar{F}((1-\delta \mu) u))$ as $u \rightarrow \infty$. But by Lemma 5.2(ii), $e^{b(u)} \mathbf{P}\left(\check{\mathcal{M}}_{u}^{c}\right) \rightarrow 0$ as $u \rightarrow \infty$, where we may take

$$
b(u)= \begin{cases}u / \log ^{2 \gamma-1} u & \text { if } F \in \operatorname{LN}(\gamma), \\ u^{1-2 \alpha} / \log u & \text { if } F \in \operatorname{WE}(\alpha) .\end{cases}
$$

Then it is easily verified that $\exp \{-b(u)\}$ decays faster than $\bar{F}((1-\delta \mu) u)$ in both the lognormal and Weibull cases (for $\alpha<1 / 3$ ).

The remainder of the proof proceeds by a truncation argument, which is identical to that used in the proof of the previous case, and a corresponding lower bound, which uses the same modifications as those just given. We omit the details, which are straightforward.

Finally, if $F \in \mathrm{WE}(\alpha)$ for $\alpha \in[1 / 3,1 / 2)$, then

$$
\mathbf{P}\left(S_{n}>v \text { for some } n \leq \delta v^{\theta} \mid X_{0}=x\right)=\mathbf{P}\left(S_{n}>u^{1 / \theta} \text { for some } n \leq \delta u \mid X_{0}=x\right) \text {. }
$$


The proof now follows as before, with the positive boundary at $u$ replaced with a boundary at $u^{1 / \theta}$. The only significant change is in the definition of $\check{m}(u)$, where $\check{a}((1-\delta \mu) u)$ is replaced with $a(u):=\check{a}\left(u^{1 / \theta}-\delta \mu u\right)$. With $\theta \in(1-\alpha, 2-3 \alpha)$ and $b(u):=u^{\{2(1-\alpha) / \theta-1\}} / \log u$, one can then verify that $\exp \{-b(u)\}$ decays faster than $\bar{F}\left(u^{1 / \theta}-\delta \mu u\right)$.

\section{Appendix}

5.1 Some auxiliary results needed in the proof of Theorem 2.2

In the following lemmas, conditions $(\mathfrak{M})$ and $(\mathfrak{D})$ will always be assumed. We begin by studying the behavior of the initial pair $\left(U_{0}, \tau_{0}\right)$.

\section{Lemma 5.1}

(i) For $\varphi$-a.a. $x$, there exists a finite constant $C_{x}$ such that

$$
\limsup _{u \rightarrow \infty} \frac{\mathbf{P}\left(U_{0}>u \mid X_{0}=x\right)}{\bar{F}(u)} \leq C_{x} .
$$

(ii) If $\mu$ is finite, then so is $\mathbf{E}\left[U_{0} \mid X_{0}=x\right]$ for $\varphi$-a.a. $x$.

(iii) If $\mathbf{E}[\exp \{\xi \tau\}]<\infty$ for some $\xi>0$, then $\mathbf{E}\left[\exp \left\{\xi \tau_{0}\right\} \mid X_{0}=x\right]<\infty$ for $\varphi$-a.a. $x$.

Proof (i) For any $K>0$, let

$$
A_{K}=\left\{x: \sup _{u \geq K} \frac{\mathbf{P}\left(U_{0}>u \mid X_{0}=x\right)}{\bar{F}(u)} \geq K\right\} .
$$

Now consider a typical regeneration cycle. According to Lemma 2.1, this begins with an initial measure of $v$ and has a duration of $\tau$; for notational convenience, we shall assume that this cycle starts at $T_{0}=1$. Define

$$
\mathcal{A}_{K}=\left\{X_{n} \in A_{K}, \text { for some } n \in[1, \tau]\right\} \quad \text { and } \quad \mathcal{O}_{L}=\left\{\min _{1 \leq n \leq \tau} S_{n} \geq-L\right\},
$$

and set $\mathfrak{T}_{A_{K}}=\inf \left\{n \geq 1: X_{n} \in A_{K}\right\}$.

If $\left\{X_{n}\right\}$ visits the set $A_{K}$ by time $\tau$, then the random variable $U$ may be decomposed into a sum of two terms, namely $U=S_{\mathfrak{T}_{A_{K}}}+\left(U-S_{\mathfrak{T}_{A_{K}}}\right)$; based on this representation, we obtain

$$
\mathbf{P}(U>u) \geq \mathbf{P}\left(\mathcal{A}_{K} \cap \mathcal{O}_{L}\right) \mathbf{P}\left(U_{0}>u+L \mid X_{0} \sim \hat{v}\right),
$$

where $\hat{v}$ is the distribution of $X_{\mathfrak{T}_{A_{K}}}$ conditioned on the event $\mathcal{A}_{K}$. By (5.2),

$$
\limsup _{u \rightarrow \infty} \frac{\mathbf{P}(U>u)}{\bar{F}(u)} \geq \limsup _{K \rightarrow \infty} \mathbf{P}\left(\mathcal{A}_{K} \cap \mathcal{O}_{L}\right) \cdot K
$$


on the right-hand side we have used the definition of $A_{K}$ and Proposition IX.1.5 of [1]. Since the left-hand side of (5.3) is finite, it follows that

$$
\mathbf{P}\left(\mathcal{A}_{K} \cap \mathcal{O}_{L}\right) \searrow 0 \quad \text { as } K \rightarrow \infty
$$

Next observe that clearly

$$
\mathbf{P}\left(\mathcal{A}_{K}\right) \leq \mathbf{P}\left(\mathcal{A}_{K} \cap \mathcal{O}_{L}\right)+\mathbf{P}\left(\mathcal{O}_{L}^{c}\right)
$$

and since the random variable $\tau$ is finite a.s.,

$$
\mathbf{P}\left(\mathcal{O}_{L}^{c}\right)=\mathbf{P}\left(\min _{1 \leq n \leq \tau} S_{n}<-L\right) \searrow 0 \quad \text { as } L \rightarrow \infty
$$

By first choosing $L$ sufficiently large and then letting $K \rightarrow \infty$, from (5.4)-(5.6) we conclude that

$$
\mathbf{P}\left(\mathcal{A}_{K}\right) \searrow 0 \text { as } K \rightarrow \infty .
$$

Hence, away from a set of measure zero, every $x \in \mathbb{S}$ belongs to some set $A_{K}^{c}$ for $K<\infty$, and consequently (5.1) holds.

(ii) Assume, to the contrary, that there exists a $\varphi$-positive set $A$ with $\mathbf{E}\left[U_{0} \mid X_{0}=x\right]$ $=\infty($ or $-\infty)$ for all $x \in A$. Let $\mathfrak{T}_{A}=\inf \left\{n: X_{n} \in A\right\}$ and observe, once again, that we may write $U=S_{\mathfrak{T}_{A}}+\left(U-S_{\mathfrak{T}_{A}}\right)$. Hence

$$
\mathbf{E}[U] \geq p\left(-L+\inf _{x \in A} \mathbf{E}\left[U_{0} \mid X_{0}=x\right]\right),
$$

where

$$
p:=\mathbf{P}\left(\mathfrak{T}_{A}<\tau \text { and } S_{\mathfrak{T}_{A}} \geq-L\right) .
$$

Since $A$ is $\varphi$-positive, $\mathbf{P}\left(\mathfrak{T}_{A}<\tau\right)>0$ ([4], Sect. 6). Hence $p>0$ for $L$ sufficiently large. Then from (5.8) it follows that $\mathbf{E}[U]=\infty$ by the choice of $A$. By [27], Lemma 5.2, $\mu=\mathbf{E}[U] / \mathbf{E}[\tau]$; hence $\mu=\infty$. This is a contradiction.

The proof of (iii) is identical.

Next we turn to a characterization of the renewal processes $N(u)$ and $M(u)$, where $N(u)-1$ denotes the random number of regenerations which occur by time $\delta u$, and $M(u)-1$ denotes the number of ladder heights occurring by this time, as formally defined in Sect. 4.

\section{Lemma 5.2}

(i) For any $\epsilon>0$, there exists a positive constant $\beta$ such that

$$
\begin{aligned}
e^{\beta u} \mathbf{P}\left(\left|N(u)-\frac{\delta u}{\mathbf{E}[\tau]}\right|>\epsilon u\right) & \rightarrow 0, \quad u \rightarrow \infty ; \\
e^{\beta u} \mathbf{P}\left(M(u)-\frac{\delta u}{\mathbf{E}[\tau] \mathbf{E}\left[\tau^{+}\right]}>\epsilon u\right) & \rightarrow 0, \quad u \rightarrow \infty .
\end{aligned}
$$


(ii) Assume that $a:[0, \infty) \rightarrow[0, \infty)$ is a function such that $a(u) / u \searrow 0$ as $u \rightarrow \infty$ and that $b:[0, \infty) \rightarrow[0, \infty)$ is a function satisfying $b(u)=o\left(a^{2}(u) / u\right)$ as $u \rightarrow$ $\infty$. Then, for any $\epsilon>0$,

$$
\begin{array}{r}
e^{b(u)} \mathbf{P}\left(\left|N(u)-\frac{\delta u}{\mathbf{E}[\tau]}\right|>\epsilon a(u)\right) \rightarrow 0, \quad u \rightarrow \infty ; \\
e^{b(u)} \mathrm{P}\left(M(u)-\frac{\delta u}{\mathbf{E}[\tau] \mathbf{E}\left[\tau^{+}\right]}>\epsilon a(u)\right) \rightarrow 0, \quad u \rightarrow \infty .
\end{array}
$$

Proof (i) is standard; see, e.g., [33], Theorem 2.3. Note that condition (D) implies that the usual large deviation moment condition is satisfied, namely,

$$
\Lambda(\xi):=\log \mathbf{E}\left[e^{\xi(\tau-\mathbf{E}[\tau])}\right]<\infty \quad \text { for some } \xi>0
$$

([20], Theorem 15.0.1). We also note that the absence of a corresponding moment condition on $\tau^{+}$implies that we only have a one-sided bound in (5.10).

The proof of (ii) is a variant of the proof of (i) and can be developed, as with (i), from Cramér's theorem ([11], Theorem 2.2.3). Let

$$
g(u)=\frac{\delta u}{\mathbf{E}[\tau]}-\epsilon a(u) .
$$

Since $N(u)$ is integer-valued, it follows by definition that $N(u)>t$ is equivalent to $T_{\lfloor t\rfloor} \leq \delta u$. Hence

$$
\mathbf{P}(N(u) \leq g(u)) \leq \mathbf{P}\left(T_{\lfloor g(u)\rfloor}>\delta u\right) \leq \mathbf{P}\left(\frac{\tilde{T}_{\lfloor g(u)\rfloor}}{\lfloor g(u)\rfloor}>h(u)\right),
$$

where $\tilde{T}_{n}=T_{n}-n \mathbf{E}[\tau]$ for all $n$, and

$$
h(u):=\frac{\epsilon \mathbf{E}[\tau](a(u)-1)}{\lfloor g(u)\rfloor} .
$$

By Cramér's theorem (and its proof),

$$
\mathbf{P}\left(\frac{\tilde{T}_{\lfloor g(u)\rfloor}}{\lfloor g(u)\rfloor}>h(u)\right) \leq C e^{-g(u) \Lambda^{*}(h(u))},
$$

where $\Lambda^{*}$ is the convex conjugate of the function $\Lambda$ defined in (5.13), and $C$ is a positive constant, where $C \rightarrow 1$ as $h(u) \rightarrow 0$. (This constant results from the inclusion of the initial term $T_{0}$, which has a distribution different from $\left\{T_{i}\right\}_{i \geq 1}$.)

Furthermore, $\Lambda$ is strictly convex, and therefore $\Lambda^{*}$ is essentially smooth ([30], Theorem 26.3). Moreover, since $\Lambda^{*}$ is a nonnegative function and the mean of $\tau-\mathbf{E}[\tau]$ is obviously zero, it follows that $\Lambda^{*}(0)=0=\left(\Lambda^{*}\right)^{\prime}(0)([11]$, Lemma 2.2.5). Thus, after a Taylor expansion, the exponent on the right-hand side of (5.15) decays as

$$
g(u)\left(\Lambda^{*}\right)^{\prime \prime}(0) \cdot \frac{(h(u))^{2}}{2} \sim \text { const } \cdot \frac{(a(u))^{2}}{u} \quad \text { as } u \rightarrow \infty
$$


by assumption, the last term grows at a faster rate than $b(u)$, thus establishing the required left-sided bound in (5.11).

The remaining estimates are obtained similarly. We omit the details.

\subsection{Proofs of Propositions 3.1 and 3.3}

Proof of Proposition 3.1 For notational convenience, assume that regeneration occurs at time one, i.e., $T_{0}=1$ in Lemma 2.1 , and let $\tau+1$ denote the subsequent regeneration time. Set

$$
\mathfrak{N}^{(\mathfrak{r})}=\mathfrak{N}_{1}+\cdots+\mathfrak{N}_{\tau}
$$

and note by [27], Lemma 5.2, that

$$
\mathbf{E}\left[\mathfrak{N}^{(\mathfrak{r})}\right]=\mathbf{E}_{\pi}[\mathfrak{N}] \mathbf{E}[\tau]=\mathbf{E}_{\pi}[\lambda(X)] \mathbf{E}[\tau],
$$

where the last step follows since $\mathfrak{N}$ has a Poisson distribution with intensity $\lambda(X)$. As $\hat{\mu}:=\mathbf{E}_{\pi}[\lambda(X)]$ and the sequence $\left\{Z_{j}^{(n)}: n \in \mathbb{Z}_{+}, j \in \mathbb{Z}_{+}\right\}$is independent of the process $\left\{\mathfrak{N}_{n}: n \in \mathbb{Z}_{+}\right\}$, it then suffices to show that

$$
\mathbf{P}\left(\sum_{k=1}^{\mathfrak{N}^{(\mathfrak{r})}} \check{Z}_{k}>u\right) \sim \mathbf{E}\left[\mathfrak{N}^{(\mathfrak{r})}\right] \bar{F}_{Z}(u) \quad \text { as } u \rightarrow \infty
$$

where $\left\{\check{Z}_{k}\right\}$ is an i.i.d. sequence of random variables with distribution $F_{Z}$.

In the regularly varying case, (5.18) follows from [14], Proposition 4.1, since, for any $\gamma<\beta$, Hölder's inequality yields

$$
\mathbf{E}\left[\left(\mathfrak{N}^{(\mathfrak{r})}\right)^{\gamma}\right] \leq \mathbf{E}\left[\tau^{\gamma}\left(\max _{1 \leq i \leq \tau} \mathfrak{N}_{i}\right)^{\gamma}\right] \leq \mathbf{E}\left[\tau^{p \gamma}\right]^{1 / p}\left(\mathbf{E}[\tau] \mathbf{E}_{\pi}\left[\mathfrak{N}^{q \gamma}\right]\right)^{1 / q}
$$

where $p^{-1}+q^{-1}=1$ and $q \gamma \leq \beta$, and where once again we have applied [27], Lemma 5.2, on the right-hand side. Moreover, the quantity on the right is finite under our hypotheses since, in particular, $(\mathfrak{D})$ implies that $\tau$ has exponential moments in a neighborhood of zero (as previously observed in (4.19)).

For the general subexponential case, (5.18) follows, provided that

$$
\mathbf{E}\left[e^{\xi Y_{\tau}}\right]<\infty \text { for some } \xi>0
$$

since by [1], Proposition IX.1.8, there would then exist a finite constant $K$ such that

$$
\begin{aligned}
& \sum_{n=0}^{\infty} \frac{\mathbf{P}\left(\sum_{k=1}^{n} \check{Z}_{k}>u\right)}{\bar{F}_{Z}(u)} \mathrm{P}\left(\mathfrak{N}^{(\mathfrak{r})}=n\right) \leq \sum_{n=0}^{\infty} K(1+\xi)^{n} \mathbf{P}\left(\mathfrak{N}^{(\mathfrak{r})}=n\right) \\
& \quad=K \mathbf{E}\left[\prod_{i=1}^{\tau} \mathbf{E}\left[(1+\xi)^{\mathfrak{N}_{i}} \mid \tau, X_{1}, \ldots, X_{\tau}\right]\right]=K \mathbf{E}\left[e^{\xi Y_{\tau}}\right]<\infty
\end{aligned}
$$

where in the last equality we have utilized the probability generating function of a Poisson random variable. This bound enables a dominated convergence argument, 
since the limit (as $u \rightarrow \infty$ ) can then be brought inside the above sum, and we may then apply Proposition IX.1.7 of [1].

To establish (5.20), let $\Lambda_{Y}(\xi)=\log \left(R(\xi)^{-1}\right)$, where $R(\xi)$ is the convergence parameter of the kernel $\hat{P}_{\xi}(x, d y):=\exp \{\xi \lambda(y)\} P(x, d y)$. (For the definition, see [29], p. 27.) Then

$$
\mathbf{E}\left[e^{\xi Y_{\tau}-\tau \Lambda_{Y}(\xi)}\right] \leq 1
$$

(This is the inequality $\hat{b}(R) \leq 1$ on p. 565 of [27]; see also Sect. 4 of that article.) Then, by Hölder's inequality,

$$
\left(\mathbf{E}\left[e^{\xi Y_{\tau}}\right]\right)^{2} \leq \mathbf{E}\left[e^{2 \xi Y_{\tau}-\tau \Lambda_{Y}(2 \xi)}\right] \mathbf{E}\left[e^{\tau \Lambda_{Y}(2 \xi)}\right] .
$$

By (5.22) the first term on the right is bounded by one. For the second term, note that $\Lambda_{Y}(\xi) \leq \Theta_{Y}(\xi)$ for all $\xi$; cf. [10], Sect. 7. Also, from the definition of $\Theta_{Y}$ it easily follows that $\Theta_{Y}(0)=0$ and that $\Theta_{Y}$ is a convex function. Consequently,

$$
\Lambda_{Y}(2 \xi) \leq \Theta_{Y}(2 \xi) \searrow 0 \quad \text { as } \xi \rightarrow 0
$$

Since (D) implies that $\tau$ has exponential moments in a neighborhood of zero (cf. (4.19)), (5.20) follows from (5.23) and (5.24).

Proof of Proposition 3.3 Without loss of generality, assume that regeneration occurs at time one, and let $\tau+1$ denote the subsequent regeneration time. Moreover, assume that regeneration is understood with respect to the joint process $\left\{\left(X_{n}, W_{n}\right)\right\}$, where $W_{n}:=\left(W_{n-1}+\log A_{n}\right)^{+}, n \in \mathbb{Z}_{+}$, and $W_{0}=0$.

First note that the Markov chain $\left\{\left(X_{n}, W_{n}\right)\right\}$ is geometrically recurrent. By [20], Theorem 15.0.1, this will be true, provided that the drift condition (D) is satisfied. Let $V(x, y)=x^{\epsilon}+e^{\epsilon y}+1, x \geq 0, y \in \mathbb{R}$, and let $C=[-K, K] \times\{0\}$, for some $K>0$. Then, for $\epsilon \leq 1$,

$$
\begin{aligned}
\mathbf{E}\left[V\left(X_{1}, W_{1}\right) \mid X_{0}=x, W_{0}=y\right] & \leq \mathbf{E}\left[\left(A_{1} x+B_{1}\right)^{\epsilon}+\left(e^{\epsilon\left(y+\log A_{1}\right)}+1\right)+1\right] \\
& \leq \mathbf{E}\left[A_{1}^{\epsilon}\right] x^{\epsilon}+\mathbf{E}\left[B_{1}^{\epsilon}\right]+\mathbf{E}\left[A_{1}^{\epsilon}\right] e^{\epsilon y}+2 \\
& =(1-\gamma) V(x, y)+\left\{\mathbf{E}\left[B_{1}^{\epsilon}\right]-\mathbf{E}\left[A_{1}^{\epsilon}\right]+2\right\}
\end{aligned}
$$

for some $\gamma>0$, provided that $\epsilon$ has been chosen sufficiently small so that $\mathbf{E}\left[A_{1}^{\epsilon}\right]<1$. This parameter $\epsilon$ can always be found, since $\Lambda_{A}^{\prime}(0)<0$ and $\Lambda_{A}$ is finite in a neighborhood of zero by assumption. Now observe that

$$
\mathbf{E}\left[B_{1}^{\epsilon}\right]-\mathbf{E}\left[A_{1}^{\epsilon}\right]+2<\frac{\gamma}{2} V(x, y) \text { for sufficiently large } K \text {. }
$$

Hence condition $(\mathfrak{D})$ is satisfied, which implies geometric recurrence.

Next observe by an inductive argument that

$$
X_{n}=\left(A_{2} \cdots A_{n}\right) X_{1}+\left(A_{3} \cdots A_{n}\right) B_{2}+\cdots+B_{n} ;
$$


consequently,

$$
\begin{aligned}
U \stackrel{d}{=} & X_{1}+\cdots+X_{\tau} \\
= & \left(1+A_{2}+A_{2} A_{3}+\cdots+A_{2} \cdots A_{\tau}\right) X_{1} \\
& +\left(1+A_{3}+\cdots+A_{3} \cdots A_{\tau}\right) B_{2}+\cdots+B_{\tau} .
\end{aligned}
$$

Let $Z_{n}:=B_{n-1} \mathbf{1}_{\{\tau \geq n-1\}}\left(1+A_{n}+A_{n} A_{n+1}+\cdots+A_{n} \cdots A_{\tau}\right), n>1$, where, for notational convenience, we have set $X_{1}=B_{1}$. Also let

$$
\mathfrak{A}:=1+A_{2}+A_{2} A_{3}+\cdots,
$$

and observe that $\mathfrak{A}$ satisfies the random recurrence equation

$$
\mathfrak{A} \stackrel{d}{=} 1+A \mathfrak{A}, \quad \text { where } A \stackrel{d}{=} A_{2}
$$

and, on the right-hand side, $A$ is independent of $\mathfrak{A}$. Since $\Lambda_{A}(\kappa)=0$ and $\Lambda_{A}$ is assumed to be finite in a neighborhood of $\kappa$, from [15], Theorem 4.1, it follows that

$$
\mathbf{P}(\mathfrak{A}>u) \leq D u^{-\kappa} \quad \text { for all } u \geq 0
$$

with some positive constant $D$. Hence, for any nonnegative random variable $W$ which is independent of $\mathfrak{A}$,

$$
\mathbf{P}(W \mathfrak{A}>u)=\mathbf{E}\left[\mathbf{P}\left(\mathfrak{A}>\frac{u}{W} \mid W\right) ; W>0\right] \leq D \mathbf{E}\left[W^{\kappa}\right] u^{-\kappa} .
$$

Moreover, by Hölder's inequality, the finiteness of $\Lambda_{B}$ in a neighborhood of zero, and the geometric recurrence of $\left\{\left(X_{n}, W_{n}\right)\right\}$,

$$
\mathbf{E}\left[B_{n-1}^{\kappa} \mathbf{1}_{\{\tau \geq n-1\}}\right] \leq \mathrm{const} \cdot \mathbf{P}(\tau \geq n-1)^{1 / 2} \leq \mathrm{const} \cdot e^{-\gamma n}
$$

for some $\gamma>0$. Finally, observe that $\left(1+A_{n}+A_{n} A_{n+1}+\cdots+A_{n} \cdots A_{\tau}\right)$ is stochastically bounded by $\left(1+A_{n}+A_{n} A_{n+1}+\cdots\right)$, and the latter quantity is equal in distribution to $\mathfrak{A}$ and independent of $B_{n-1} \mathbf{1}_{\{\tau \geq n-1\}}$. Hence (5.26) and (5.27) yield

$$
\mathbf{P}\left(Z_{n}>u\right) \leq D^{\prime} u^{-\kappa} \cdot e^{-\gamma n} \quad \text { for some } D^{\prime}>0 .
$$

Letting $\beta=e^{\gamma / 2 \kappa}$ and $K=\sum_{n=0}^{\infty} \beta^{-n}$ then gives

$$
\mathbf{P}\left(\sum_{n=k}^{\infty} Z_{n}>u\right) \leq \sum_{n=k}^{\infty} \mathbf{P}\left(Z_{n}>\frac{\beta^{-n}}{K} u\right) \leq \Delta(k) u^{-\kappa}
$$

where

$$
\Delta(k):=D^{\prime} K^{\kappa} \sum_{n=k}^{\infty} e^{-\gamma n / 2} \rightarrow 0 \quad \text { as } k \rightarrow \infty .
$$


It remains to study the asymptotic decay of $\sum_{n=2}^{k-1} Z_{n}$. From the definition of $\left\{Z_{n}\right\}$ we obtain, after a short computation,

$$
\sum_{n=2}^{k-1} Z_{n}=\left(B_{1} A_{2} \cdots A_{k-1}+\cdots+B_{k-1}\right) \mathfrak{A}_{k}+\mathfrak{B}_{k}
$$

where $\mathfrak{A}_{k}:=\left(1+A_{k}+A_{k} A_{k+1}+\cdots+A_{k} \cdots A_{\tau}\right) \mathbf{1}_{\{\tau \geq k-1\}}$;

$$
\begin{aligned}
\mathfrak{B}_{k}:= & \left(1+A_{2} \mathbf{1}_{\{\tau \geq 2\}}+A_{2} A_{3} \mathbf{1}_{\{\tau \geq 3\}}+\cdots+A_{2} \cdots A_{k-2} \mathbf{1}_{\{\tau \geq k-2\}}\right) B_{1} \\
& +\left(\mathbf{1}_{\{\tau \geq 2\}}+A_{3} \mathbf{1}_{\{\tau \geq 3\}}+\cdots+A_{3} \cdots A_{k-2} \mathbf{1}_{\{\tau \geq k-2\}}\right) B_{2}+\cdots+B_{k-2} \mathbf{1}_{\{\tau \geq k-2\}} .
\end{aligned}
$$

Observe that, by Minkowski's inequality (or Eq. (9.26) of [15] if $\kappa+\epsilon<1$ ), $\mathbf{E}\left[\mathfrak{B}_{k}^{\kappa+\epsilon}\right]<\infty$ for some $\epsilon>0$. Hence Chebyshev's inequality yields that, for any fixed $k$,

$$
\mathbf{P}\left(\mathfrak{B}_{k}>u\right) \leq \text { const } \cdot u^{-(\kappa+\epsilon)} .
$$

Thus, $\mathfrak{B}_{k}$ is negligible relative to terms which decay as $u^{-\kappa}$.

Next, consider the asymptotic decay of $\mathfrak{A}_{k}$. Note that

$$
\begin{aligned}
\mathfrak{V}_{k} \mathbf{1}_{\{\tau \geq k-1\}}:= & \left(1+A_{k}+A_{k} A_{k+1}+\cdots\right) \mathbf{1}_{\{\tau \geq k-1\}} \\
= & \left(1+A_{k}+A_{k} A_{k+1}+\cdots+A_{k} \cdots A_{\tau}\right) \mathbf{1}_{\{\tau \geq k-1\}} \\
& +\left(A_{k} \cdots A_{\tau+1}\right)\left(1+A_{\tau+2}+A_{\tau+2} A_{\tau+3}+\cdots\right) \mathbf{1}_{\{\tau \geq k-1\}} \\
:= & \mathfrak{A}_{k}+\left(A_{k} \cdots A_{\tau+1}\right) \hat{\mathfrak{A}} \mathbf{1}_{\{\tau \geq k-1\}}
\end{aligned}
$$

where $\mathfrak{V}_{k} \stackrel{d}{=} \mathfrak{A}$ and $\hat{\mathfrak{A}} \stackrel{d}{=} \mathfrak{A}$. Now $A_{k} \cdots A_{\tau}<1$, since, by construction, regeneration occurs after a time of decrease of the process $\left\{\log A_{n}\right\}$, that is, one of its negative ladder height times. (This is because the small set used above to derive $(\mathfrak{D})$ was taken to be $C=[-K, K] \times\{0\}$, implying $W_{\tau}=0$.) Moreover, by the regeneration construction (described, e.g., in [26]), we also have that $A_{\tau+1}$ is independent of $\left(A_{k} \cdots A_{\tau}\right)$ and of $\hat{\mathfrak{A}}$ and - for an appropriate choice of the minorization measure $v$ in $(\mathfrak{M})$ - also bounded from above by one. Moreover, by [15], Theorem 4.1,

$$
\mathbf{P}(\mathfrak{A}>u) \sim D^{\prime \prime} u^{-\kappa} \quad \text { as } u \rightarrow \infty .
$$

Since $\mathfrak{A} \stackrel{d}{=} \hat{\mathfrak{A}}$ (where the right-hand side is conditional on $\{\tau \geq k-1\}$ ), from a result of Breiman (as given in [19], Lemma 2.2) it follows that

$$
\mathbf{P}\left(\left(A_{k} \cdots A_{\tau+1}\right) \mathbf{1}_{\{\tau \geq k-1\}} \hat{\mathfrak{A}}>u\right) \sim \gamma_{k} D^{\prime \prime} u^{-\kappa} \quad \text { as } u \rightarrow \infty
$$

where $\gamma_{k}:=\mathbf{E}\left[\left(A_{k} \cdots A_{\tau+1}\right)^{\kappa} \mathbf{1}_{\{\tau \geq k-1\}}\right]<\mathbf{P}(\tau \geq k-1)$. Since $\mathfrak{V}_{k} \stackrel{d}{=} \mathfrak{A}$ and is independent of $\mathbf{1}_{\{\tau \geq k-1\}}$, from (5.32), (5.33), (5.34), and an elementary argument it follows that

$$
\mathbf{P}\left(\mathfrak{A}_{k}>u\right) \sim D^{(k)} u^{-\kappa} \quad \text { as } u \rightarrow \infty
$$

for some constant $D^{(k)}>0$.

Springer 
Applying Breiman's result once again (cf. (5.34)), but now to the quantity on the right-hand side of (5.30), we obtain

$$
\mathbf{P}\left(\sum_{n=2}^{k-1} Z_{n}>u\right) \sim C_{k} u^{-\kappa} \quad \text { as } u \rightarrow \infty
$$

where $C_{k}:=\mathbf{E}\left[Y_{k}^{\kappa}\right]$ and $Y_{k}:=D^{(k)}\left(B_{1} A_{2} \cdots A_{k-1}+\cdots+B_{k-1}\right)$. Moreover, by Minkowski's inequality we have that $C_{k}<\infty$ for any given $k$. We conclude

$$
\mathbf{P}\left(\sum_{n=2}^{k-1} Z_{n}\right) \sim C_{k} u^{-\kappa} \quad \text { and } \quad \mathbf{P}\left(\left(U-\sum_{n=2}^{k-1} Z_{n}\right)>u\right) \leq \Delta(k) u^{-\kappa}
$$

where $\Delta(\kappa) \rightarrow 0$ as $k \rightarrow \infty$. It follows that $\mathbf{P}(U>u) \sim C u^{-\kappa}$, where $C:=\lim _{k \rightarrow \infty} C_{k}$. This constant must be finite because, for any fixed $k$, $C \leq$ const $\cdot\left(C_{k}+\Delta(k)\right)$. Moreover, it must be positive because $C_{k}>0$ and the lefthand side of (5.36) is a lower bound for $\mathbf{P}(U>u)$.

\section{References}

1. Asmussen, S.: Ruin Probabilities. World Scientific, River Edge (2000)

2. Asmussen, S., Henriksen, L.F., Klüppelberg, C.: Large claims approximations for risk processes in a Markovian environment. Stoch. Process. Appl. 54, 29-43 (1994)

3. Asmussen, S., Schmidli, H., Schmidt, V.: Tail probabilities for non-standard risk and queuing processes with subexponential jumps. Adv. Appl. Probab. 31, 422-447 (1999)

4. Athreya, K.B., Ney, P.: A new approach to the limit theory of recurrent Markov chains. Trans. Am. Math. Soc. 245, 493-501 (1978)

5. Bahadur, R.R.: Some Limit Theorems in Statistics. SIAM, Philadelphia (1971)

6. Basel Committee on Banking Supervision: Consultative document. Overview of the new Basel capital accord. http://www.bis.org/publ/bcbsca01.pdf (2001)

7. Basrak, B., Davis, R.A., Mikosch, T.: Regular variation of GARCH processes. Stoch. Process. Appl. 99, 95-115 (2002)

8. Bollerslev, T.: Generalized autoregressive conditional heteroskedasticity. J. Econom. 31, 307-327 (1986)

9. Crouhy, M., Galai, D., Mark, R.: Risk Management. McGraw-Hill, New York (2000)

10. de Acosta, A.: Large deviations for vector-valued functionals of a Markov chain: lower bounds. Ann. Probab. 16, 925-960 (1988)

11. Dembo, A., Zeitouni, O.: Large Deviations Techniques and Applications, 2nd edn. Springer, Berlin (1998)

12. Embrechts, P., Kaufmann, R., Samorodnitsky, G.: Ruin theory revisited: stochastic models for operational risk. In: Bernadell, C., et al. (eds.) Risk Management for Central Bank Foreign Reserves, pp. 243-261. European Central Bank, Frankfurt a.M. (2004)

13. Embrechts, P., Klüppelberg, C., Mikosch, T.: Modelling Extremal Events. Springer, Berlin (1997)

14. Faÿ, G., González-Arávalo, B., Mikosch, T., Samorodnitsky, G.: Modeling teletraffic arrivals by a Poisson cluster process. Queuing Syst. Appl. 54, 121-140 (2006)

15. Goldie, C.M.: Implicit renewal theory and tails of solutions of random equations. Ann. Appl. Probab. 1, 126-166 (1991)

16. Hamilton, J.D.: A new approach to the economic analysis of nonstationary time series and the business cycle. Econometrica 57, 357-384 (1989)

17. Hardy, M.R.: A regime-switching model of long-term stock returns. North Am. Actuar. J. 5, 41-53 (2001)

18. Höing, A.: Topics in risk management for insurance and finance: ruin and dependence. PhD dissertation, Mathematische Wissenschaften, Swiss Federal Institute of Technology, Zürich, Nr. 16128. http://e-collection.ethbib.ethz.ch/diss/alph/h.html (2005) 
19. Konstantinides, D., Mikosch, T.: Large deviations and ruin probabilities for solutions to stochastic recurrence equations with heavy-tailed innovations. Ann. Probab. 33, 1992-2035 (2005)

20. Meyn, S.P., Tweedie, R.L.: Markov Chains and Stochastic Stability. Communications and Control Engineering Series. Springer, London (1993)

21. Mikosch, T.: Modeling dependence and tails of financial time series. In: Finkenstädt, B., Rootzén, H. (eds.) Extreme Values in Finance, Telecommunications, and the Environment, pp. 185-286. Chapman and Hall, Boca Raton (2003)

22. Mikosch, T., Nagaev, A.V.: Large deviations of heavy-tailed sums with applications in insurance. Extremes 1, 81-110 (1998)

23. Nagaev, A.V.: Integral limit theorems for large deviations when Cramér's condition is not fulfilled I. Theory Probab. Appl. 14, 51-64 (1969)

24. Nagaev, A.V.: Integral limit theorems for large deviations when Cramér's condition is not fulfilled II. Theory Probab. Appl. 14, 193-208 (1969)

25. Nagaev, S.V.: On the asymptotic behavior of probabilities of one-sided large deviations. Theory Probab. Appl. 26, 369-372 (1981)

26. Ney, P., Nummelin, E.: Some limit theorems for Markov additive processes. In: Janssen, J. (ed.) Semi-Markov Models. Theory and Applications. Proceedings of an International Symposium on SemiMarkov Processes and their Applications Held in Brussels, June 1984, pp. 3-12. Plenum, New York (1984)

27. Ney, P., Nummelin, E.: Markov additive processes I. Eigenvalue properties and limit theorems. Ann. Probab. 15, 561-592 (1987)

28. Nummelin, E.: A splitting technique for Harris recurrent Markov chains. Z. Wahrsch. Gebiete 43, 309-318 (1978)

29. Nummelin, E.: General Irreducible Markov Chains and Nonnegative Operators. Cambridge Tracts in Mathematics, vol. 83. Cambridge University Press, Cambridge (1984)

30. Rockafellar, R.T.: Convex Analysis. Princeton University Press, Princeton (1970)

31. Rogers, L.C.G., Zane, O.: Designing and estimating models of high frequency data. http://www. statslab.cam.ac.uk/chris/papers.html/tick.pdf (1998)

32. Rydberg, T.H., Shephard, N.: A modelling framework for the prices and times of trades made on the New York stock exchange. In: Walden, A.T., Fitzgerald, W.J., Smith, R.L., Young, P.C. (eds.) Nonlinear and Nonstationary Signal Processing, pp. 217-246. Cambridge University Press, Cambridge (2000)

33. Tiefeng, J.: Large deviations for renewal processes. Stoch. Process. Appl. 50, 57-71 (1994) 